\title{
Arille de Blighia sapida (K. Koenig, 1778): potentialités nutritionnelles, effets toxiques et carentiels liés à sa consommation, selon le stade de récolte et le degré de consommation
}

\author{
Howélé OUATTARA *, Alassane MEITE, Augustin K. AMONKAN, \\ Koffi G. KOUAME et Séraphin KATI-COULIBALY \\ Laboratoire de Nutrition Pharmacologie, UFR, Biosciences, Université de Cocody-Abidjan, \\ Côte d'Ivoire. \\ "Auteur correspondant,E-mail : ouattara_howele@yahoo.fr
}

\section{RESUME}

Le fisanier (Blighia sapida K. Koenig) est une plante originaire d'Afrique occidentale. L'arille du fruit est la partie la plus utilisée de la plante. Il se consomme cru, bouilli, ou frit. Il est même consommé dans certaines situations particulières pour pallier l'absence de nourriture. Les études menées sur l'arille indiquent sa richesse en lipides, en protéines, en vitamines $C$ et en éléments minéraux. L'huile extraite de l'arille est utilisée pour les grillades et pourrait être aussi utilisée en cosmétique et dans l'industrie pharmaceutique. Du fait de la présence de molécules potentiellement toxiques, l'usage populaire recommande qu'on le consomme à maturité et/ou après séchage au soleil. En effet, dans ces conditions, la teneur de l'hypoglycine A, molécule toxique, est fortement réduite dans le fruit. Les risques de toxicité sont liés au fait que l'hypoglycine A bloque la $\beta$ oxydation et entraîne ainsi une hypoglycémie pouvant conduire à la mort. Malgré la relative toxicité de l'arille selon la période de récolte, il demeure une source nutritionnelle importante et donc un élément dont il faut tenir compte dans l'alimentation des peuples de certaines régions tropicales.

(C) 2011 International Formulae Group. All rights reserved.

Mots clés: Arille, alimentation, toxique.

\section{INTRODUCTION}

Les régimes alimentaires des populations d'une aire géographique et culturelle donnée est le résultat de la disponibilité des espèces animales et végétales, et des expériences liées à leur consommation en tant qu'aliment.

De nombreux régimes de base traditionnelle sont fondés sur les potentialités nutritionnelles et thérapeutiques qu'un produit alimentaire évoque.
De nombreux interdits alimentaires ont pour origine l'observation d'effets toxiques consécutifs à la consommation d'un aliment. L'incapacité des populations à donner une explication scientifique, et donc d'établir un lien de causalité entre la présence d'une substance toxique ou un mauvais usage en fait un aliment tabou. Blighia sapida (BHS) qui est un fruit connu dans les zones de l'Afrique occidentale et des caraïbes (Jamaïque, Haïti), est riche en protéines (Morton, 1987; Akintayo et al., 2002). Cependant, plusieurs 
accidents liés à sa consommation ont été signalés (CDC, 1992 ; Meda et al., 1999 ; Organisation Panaméricaine de la Santé, 2002 ; Edleston et Person, 2003).

La présente étude a pour but d'analyser les connaissances établies sur les propriétés de l'arille de Blighia sapida et de comprendre les mécanismes qui pourraient expliquer les effets toxiques signalés.

\section{PRESENTATION DE LA PLANTE Classification}

Le fisanier appartient au règne des plantae, à la division des Magnoliaphyta et à l'ordre des sapindales. Le nom binomial de cette plante est Blighia sapida. La plante a été décrite par K. D. Koenig en 1782.

\section{Historique}

Le genre Blighia a été nommé en l'honneur du capitaine William Bligh, un marin anglais qui, en 1789 , reçut pour mission de rapporter l'arbre à pain (Artrocarpus altilis) depuis l'archipel malais jusqu'en Amérique du Sud. Le voyage a été rendu célèbre dans le monde entier par la mutinerie de la Bounty (Blancke, 2001). C'est également au cours de ce voyage qu'il transporta ce fruit de la Jamaïque en Angleterre (Lewis, 1965).

\section{Description}

C'est un arbre persistant, élancé, à couronne buissonnante pouvant atteindre jusqu'à $20 \mathrm{~m}$ de haut.

Les feuilles sont alternes, composées et possèdent 6 à10 folioles opposées. Les fleurs réunies en grappes axillaires sont blanches crèmes, très odorantes et mesurent de 4 à $5 \mathrm{~mm}$.

Les fruits piriformes possèdent trois loges renflées dont le diamètre est compris entre 7 et $8 \mathrm{~cm}$. A maturité, ils virent du rose au jaune, puis s'ouvrent par trois valves laissant découvrir trois graines noires et brillantes adhérant à un arille charnu jaune clair (Blancke, 2001).

\section{Distribution}

Cette espèce est originaire d'Afrique occidentale. Elle a été répandue par la culture dans toutes les régions tropicales. Elle prospère surtout dans les régions chaudes et humides, à basses altitudes jusqu'à $700 \mathrm{~m}$ d'altitude (Arbonnier, 2002).

\section{Utilisation}

Les arilles des fruits mûrs et ouverts sont comestibles; c'est d'ailleurs un fruit très répandu. Le moment de récolte est particulièrement critique : les fruits immatures ou cueillis trop tôt sont très toxiques, ainsi que les graines. Chaque année, on recense de nombreux cas d'empoisonnements mortels. Les arilles sont exportés en conserves. A la Jamaïque, ces fruits savoureux, accompagnent les plats de poissons (Blancke, 2001).

Les fruits non mûrs écrasés produisent la mousse qui est employée comme savon. Des extraits de graisses sont employés dans le traitement des parasites. Le fruit mûr est consommé pour abaisser la fièvre et pour maîtriser la dysenterie. La peau externe du fruit est utilisée pour soigner les panaris.

Un poultice de feuilles écrasées est appliqué au front et à la peau pour alléger les maux de tête, les ulcères, la conjonctivite, l'angine, l'ictère et les vertiges.

Le bois termite résistant est utilisé localement comme matériau de construction et comme bois de feu (Arbonnier, 2002).

\section{COMPOSITION DE L'ARILLE}

Les lipides sont les constituants majoritaires de l'arille de BHS. En effet, les différentes analyses de l'arille révèlent des taux de lipides allant de 44,22\% à 45,50\% (Morton, 1987; Akintayo et al., 2002). Cette proportion d'huile est comparable à celle contenue dans l'arachide qui est de 45,50\% (Food Agriculture Organisation, 2000).

Deux études ont révélé une quantité importante de protéines dans l'arille de BHS. Selon Morton (1987), l'arille de BHS renfermerait $20,64 \%$ de protéines. L'étude réalisée par Akintayo et al. (2002) confirme cette assertion sur le taux élevé de protéines, 
avec une teneur de $24,30 \%$ dans l'arille de BHS séché à l'étuve à $50 \pm 5^{\circ} \mathrm{C}$.

Les études ont montré que l'arille de BHS contient une faible quantité d'hydrates de carbone. Cette valeur serait selon Morton (1987) de 9,55\% et selon Akintayo et al. (2002) de 6,53\%.

Les fibres représentent selon Morton (1987), 3,45\% de l'arille, et selon Akintayo et al. (2002), 4,23\% de l'arille.

Les études effectuées indiquent une proportion élevée d'acide ascorbique (65mg/kg) dans l'arille de BHS. L'arille contient également de la thiamine $(0,1 \mathrm{mg} / \mathrm{kg})$, de la niacine $(3,74 \mathrm{mg} / \mathrm{kg})$, de la riboflavine $(0,18 \mathrm{mg} / \mathrm{kg})$ et aussi des traces de caroténoïdes. La présence de fortes quantités d'huile dans l'arille de BHS militerait en faveur d'une proportion appréciable de vitamines liposolubles (A, D, E et K) dans cet arille.

Les minéraux de l'arille de BHS représentent 4,41\% de l'arille selon Morton (1987). Les travaux de Akintayo et al. (2002) donnent une valeur de cendres de 5,60\%.

Les différents travaux montrent que l'arille de BHS contient du calcium (83 $\mathrm{mg} / \mathrm{kg}$ ), du phosphore (98mg/kg), du fer $(5,52 \mathrm{mg} / \mathrm{kg})$, du sodium $(124 \mathrm{mg} / \mathrm{kg}), \mathrm{du}$ magnésium $(271 \mathrm{mg} / \mathrm{kg})$, du cuivre $(8,1 \mathrm{mg} / \mathrm{kg})$ et $\mathrm{du}$ zinc $(6,5 \mathrm{mg} / \mathrm{kg})$ (Morton, 1987 ; Akintayo et al. (2002)).

Le Tableau 1 donne la composition nutritionnelle de l'arille de BHS préparé. Le Tableau 2 donne la composition nutritionnelle de l'arille séché à l'étuve à $50 \pm 5^{\circ} \mathrm{C}$ pendant 48 heures.

\section{VALEURS NUTRITIONNELLES}

L'arille de Blighia sapida (BHS) est consommé cru, bouilli, frit ou en sauce (Arbonnier, 2002). Déjà en 1948, dans : « Note sur la valeur alimentaire de BHS», puis en 1958, dans «Rapport Institut Pasteur AOF », Auffret et Tanguy affirment qu'agréablement consommé en marge de repas, ce fruit constitue par sa composition en matière grasse, protides et vitamine $\mathrm{C}$, un élément important voire un atout à ne pas négliger au cours de l'appréciation mathématique de la ration alimentaire de nombreux peuples.

Cette valeur nutritionnelle pourrait être appréciée par sa proportion d'huile comparable à celle contenue dans l'arachide qui est de 45,50\% (Food Agriculture Organisation, 2000). Elle s'apprécierait aussi par la proportion des différents acides gras qui y sont représentés. Selon Omobuwajo et al. (2000), l'acide linoléique, l'acide palmitique et l'acide stéarique seraient les plus grands constituants de l'huile extraite des graines du fruit de BHS. Une étude récente menée au Bénin indique que l'huile extraite des graines $\mathrm{du}$ fruit de BHS renferme une haute proportion $(63,8 \%)$ d'acides gras monoinsaturés avec une proportion intéressante $(48,4 \%)$ d'acides éicosanoïques (Djenotin et al., 2009). La composition en acides gras des graines ne serait vraisemblablement pas éloignée de celle de l'arille. A se sujet, une étude très récente menée au Nigeria donne les proportions d'acides gras présentes dans l'huile extraite de l'arille de BHS suivante: 22,22\% d'acides gras saturés, $56,43 \%$ d'acides gras monoinsaturés et $21,35 \%$ d'acides gras polyinsaturés. Cette huile selon Oladiji et al. (2009) est plus riche en acides béhéniques, palmitoléiques, oléiques, gadoléiques, éruciques et 9, 12 éicosanoique que l'huile de soja. Le pourcentage d'élévation de ces gras par rapport aux proportions contenus dans l'huile de soja sont respectivement de $15,75 \%$, $0,89 \%, 7,22 \%, 12,05 \%, 8,27 \%$ et $21,35 \%$ (Oladiji et al., 2009).

Le fait que cette huile soit riche en acides gras monoinsaturés et en acides gras polyinsaturés, notamment l'acide linoléique, un acide gras essentiel, constituerait un atout important pour l'utilisation de cette huile en alimentation. Malgré les vertus nutritionnelles avérées, la recommandation industrielle (cosmétiques, pharmaceutiques) serait la mieux indiquée car des travaux indiquent des effets délétères liés à l'utilisation expérimentale d'huile extraite de l'arille de BHS. En effet, Mcintosch (1971) a montré 
que l'huile extraite de l'arille immature induit une toxicité rénale chez le rat. Aussi, d'après Singh et al. (1992), des injections intrapéritonéales d'huile d'arille de BHS récoltés immatures induisent des activités hypertensives aux rats.

D'après les différentes études, la proportion de protéines dans l'arille de BHS n'est pas éloignée de celle de l'arachide qui est de 27\% (Food Agriculture Organisation, 2000). Cette proportion de protéines est plus élevée par rapport aux recommandations officielles qui se situent entre 12 et $15 \%$ (Food Agriculture Organisation, 2003). Si la disponibilité des protéines dans une diète constituée essentiellement de l'arille de BHS n'est vraisemblablement pas un facteur limitant, elle pourrait être entravée par la présence de L'hypoglycine $\mathrm{A}$, un aminopeptide à effet hypoglycémiant, si l'arille est récolté immature (Organisation Panaméricaine de la Santé, 2002). Il convient de le récolter à pleine maturité, lorsqu'il s'ouvre seul, sous l'effet du rayonnement solaire. A défaut, dans le cas exceptionnel de pénurie de nourriture, l'exposer au soleil, afin d'éliminer une bonne partie de la molécule toxique (Organisation Panaméricaine de la Santé, 2002).

Il est établi que les hydrates de carbone devraient constituer la part majoritaire des besoins énergétiques de l'Homme (Food Agriculture Organisation/World Heath Organisation, 1998). La disponibilité des hydrates de carbone dans l'arille de BHS étant faible, le rôle énergétique d'une diète essentiellement à base d'arille de BHS est donc en grande partie assuré par les lipides. Cela pourrait élever le facteur de risque d'athérosclérose et donc de maladies cardiovasculaires.

Les proportions de fibres sont comparables à celles préconisées par l'Institut Américain de Nutrition (Reeves et al., 1993). Cette proportion de fibres est donc suffisante pour garantir une bonne digestibilité aux consommateurs de l'arille de BHS. De plus, cette bonne proportion de fibres pourrait militer en faveur d'une diminution des taux plasmatiques de mauvais cholestérol «cholestérol-LDL» dans le cas des diètes essentiellement constituées de l'arille de BHS.

Les études effectuées indiquent une diversité de vitamines (acides ascorbiques, thiamine, niacine, riboflavine et aussi des traces de caroténoïdes). La présence de fortes quantités d'huile dans l'arille de BHS milite en faveur d'une proportion appréciable de vitamines liposolubles (A, D, E et K). Dans le cas d'une diète essentiellement constituée de l'arille de BHS, le facteur vitaminique limitant relaté par l'Organisation Panaméricaine de la Santé, serait lié à une carence en quelques vitamines hydrosolubles.

La quantité de minéraux dans l'arille de BHS est équivalente à la valeur minérale du poids corporel. Elle est également, selon les différents résultats des analyses, bien diversifiées. Ceci suggère que la fraction minérale de l'arille de BHS serait suffisante pour assurer les rôles biologique et physiologique qu'on confère aux minéraux.

\section{EFFETS TOXICOLOGIQUES ET CARENTIELS}

Malgré les valeurs nutritionnelles avérées de l'arille de BHS, il demeure une source d'intoxication redoutable. Il est toxique surtout lorsqu'il est consommé à un stade précoce de maturation (Hassall et Reyle, 1955). Dans ce cas, les causes d'intoxications sont dues à une molécule toxique appelée hypoglycine $\mathrm{A}$, un aminopeptide à effet hypoglycémiant (Hassall et Reyle, 1954), qui se trouve à des proportions très élevées dans le fruit immature $(1000 \mathrm{ppm})$ et très faibles dans le fruit mûr ( $<100 \mathrm{ppm})$ (Chase et al., 1990 ; Brown et al., 1992). Ces intoxications sont généralement observées chez les enfants qui consomment accidentellement des quantités élevées d'arille immature (Edleston et Person, 2003).

La consommation à des degrés élevés de l'arille de BHS afin de pallier l'absence de nourriture peut provoquer des avitaminoses, notamment chez les enfants, aboutissant ainsi à des décès (Organisation Panaméricaine de la Santé, 2002). 
Plusieurs cas de morbidité dus à la consommation de l'arille de BHS ont été recensés à travers le monde :

- En 1984 plus de 100 décès, principalement des enfants, ont été recensés en Côte d'Ivoire (département de Katiola);

- Entre 1989 et 1991, des cas de morts ont été relatés en Jamaïque (CDC, 1992) ;

- Entre 1996 et 1997, des cas de décès ont été recensés au Burkina-Faso (Meda et al., 1999); - Entre 1998 et 2000, le nord d'Haïti a été frappé par une catastrophe naturelle (inondation), ce qui a eu pour conséquence, une diminution de la production agricole. Il a été suggéré que l'élévation de la consommation de l'arille de BHS a été la principale cause de la mort de plus de 100 personnes (Organisation Panaméricaine de la Santé, 2002).

De tous les cas ci-dessus énumérés, les enfants demeurent les plus vulnérables à cette intoxication et à la carence nutritionnelle liée à la consommation importante de l'arille de BHS. Les causes de décès liées aux avitaminoses et aux carences nutritionnelles sont rares et surviennent en cas de famine (CDC, 1992 ; Meda et al., 1999 ; Organisation Panaméricaine de la Santé, 2002).

\section{MECANISMES D'ACTION DE LA MOLECULE TOXIQUE}

Plusieurs travaux ont permis d'élucider le mécanisme d'action de la molécule toxique. Hassall et Reyle (1955) utilisent une méthode d'extraction qui leur a permis d'isoler et de purifier cette molécule (hypoglycine A).

Les travaux de Von Holt et Bohn (1966), Tanaka (1975) et Kean (1976) ont permis de démontrer que cette toxine, hypoglycine $\mathrm{A}$, ingérée, est métabolisée dans le foie en un métabolite actif, le Méthylène Cyclopropyl Acétyl Coenzyme A (MCPCOA) qui serait à l'origine du coma hypoglycémique.

Manchester (1974), puis Kean (1976) démontrent respectivement que cette toxine hépatique inhibe l'oxydation des acides gras, $\beta$-oxydation, aussi bien in vivo que in vitro. Alexandra et al. (1981) aboutissent au fait que le métabolite actif, MCPCOA, lie de manière irréversible la Flavine Adénine Dinucléotide (FAD), forme active de la riboflavine (vitamine B2), bloquant ainsi les courtes et moyennes chaînes d'acyl coenzyme déshydrogénase puisque la FAD en est le coenzyme.

Le coma hypoglycémique observé est donc dû à un catabolisme anormalement exagéré des hydrates de carbones puisque les lipides ne constituent quasiment plus une source d'énergie. Les travaux de Ming-Tai et al. (1991, 1992, 1993) indiquent qu'en cas d'intoxication à l'hypoglycine $\mathrm{A}$, les taux plasmatiques de lipides augmentent ainsi que les taux urinaires d'urée et d'aminoacides. Le mécanisme général d'action de l'hypoglycine A et de ses dérivés est donné par la Figure 1.

\section{TRAITEMENT ANTIDOTIQUE DE L'INTOXICATION A L'HYPOGLYCINE A}

La recherche d'antidote à l'hypoglycine A n'a pas encore donné de résultats à efficacités absolues. Cependant, certains travaux de chercheurs méritent d'être notés. L'équipe de Chercheurs du Centre Muraz de Bobo-Dioulasso (Burkina Faso) a démontré qu'à défaut d'un traitement efficace contre l'hypoglycine A, que l'injection de glucose retarderait la mort chez la souris plus que l'association bleu de méthylène glucose, qui elle même retarderait la mort plus que le bleu de méthylène employé seul (Barennes et al., 2004). L'administration de sucre et surtout de glucose est donc recommandée en cas d'intoxication liée à l'ingestion d'arille de BHS. Il est également recommandé afin d'éviter les vomissements, les contractions gastrointestinales, d'utiliser des antiémétiques. L'administration de riboflavine (vitamine B2) et de glycine est reconnue comme antagonisant les effets toxiques de l'hypoglycine A (Goldson, 2011). En effet, des allégations suggèrent que la riboflavine (vitamine B2) stimulerait de nouveau la synthèse d'acyl coenzyme A déshydrogénase alors que l'injection de glycine en même temps que la réalisation des processus de $\beta$ - 
oxydation augmenterait le métabolisme lipidique initialement défaillant (Al-Bassam et Sherratt, 1981).

\section{AUTRES METABOLITES CONTENUS} DANS LE FRUIT DE BLIGHIA SAPIDA

D'autres métabolites ont été isolés du fruit de Blighia sapida. Bien qu'ils ne possèdent pas des propriétés biologiques intéressantes, les structures de ces métabolites sont naturellement inhabituelles :

- Blighione est une grosse quinone modérément soluble qui a été isolée de l'arille de BHS (Garg et Mitra, 1968);

- Vomifoliol est une molécule également présente dans l'arille de BHS. Elle serait impliquée dans la régulation de l'ouverture du fruit à maturité (Stuart et al., 1976) ;

Tableau 1: Composition nutritionnelle approximative pour $100 \mathrm{~g}$ d'arille de BHS préparé.

\begin{tabular}{lc}
\hline Paramètre & Pourcentage pour 100 g \\
Humidité & $57,60 \mathrm{~g}$ \\
Protéines & $8,75 \mathrm{~g}$ \\
Lipides & $18,78 \mathrm{~g}$ \\
Fibres & $3,45 \mathrm{~g}$ \\
Hydrates de carbone & $9,55 \mathrm{~g}$ \\
Cendres & $1,87 \mathrm{~g}$ \\
Calcium & $83 \mathrm{mg}$ \\
Phosphore & $98 \mathrm{mg}$ \\
Fer & $5,52 \mathrm{mg}$ \\
Carotène & $\ldots \ldots \ldots$ \\
Thiamine & $0,10 \mathrm{mg}$ \\
Riboflavine & $0,18 \mathrm{mg}$ \\
Niacine & $3,74 \mathrm{mg}$ \\
Acide ascorbique & $65 \mathrm{mg}$ \\
\hline \multicolumn{2}{c}{ Source: Morton (1987) }
\end{tabular}

Tableau 2: Composition nutritionnelle approximative pour $100 \mathrm{~g}$ d'arille de BHS séché à l'étuve à $50^{\circ} \mathrm{C}$ pendant 24 heures.

\begin{tabular}{lc}
\hline Paramètre & Teneur pour 100 g \\
Humidité & $13,80 \mathrm{~g}$ \\
Protéines & $24,30 \mathrm{~g}$ \\
Lipides & $45,50 \mathrm{~g}$ \\
Fibres & $4,23 \mathrm{~g}$ \\
Hydrates de carbone & $6,53 \mathrm{~g}$ \\
Cendres & $5,60 \mathrm{~g}$ \\
Calcium & $32,6 \mathrm{mg}$ \\
Phosphore & $240 \mathrm{mg}$ \\
Fer & \multicolumn{2}{c}{} \\
Potassium & $17,5 \mathrm{mg}$ \\
Magnésium & $951 \mathrm{mg}$ \\
Cuivre & $271 \mathrm{mg}$ \\
Zinc & $8,1 \mathrm{mg}$ \\
\multicolumn{1}{c}{ Source : Akintayo et al. (2002) }
\end{tabular}



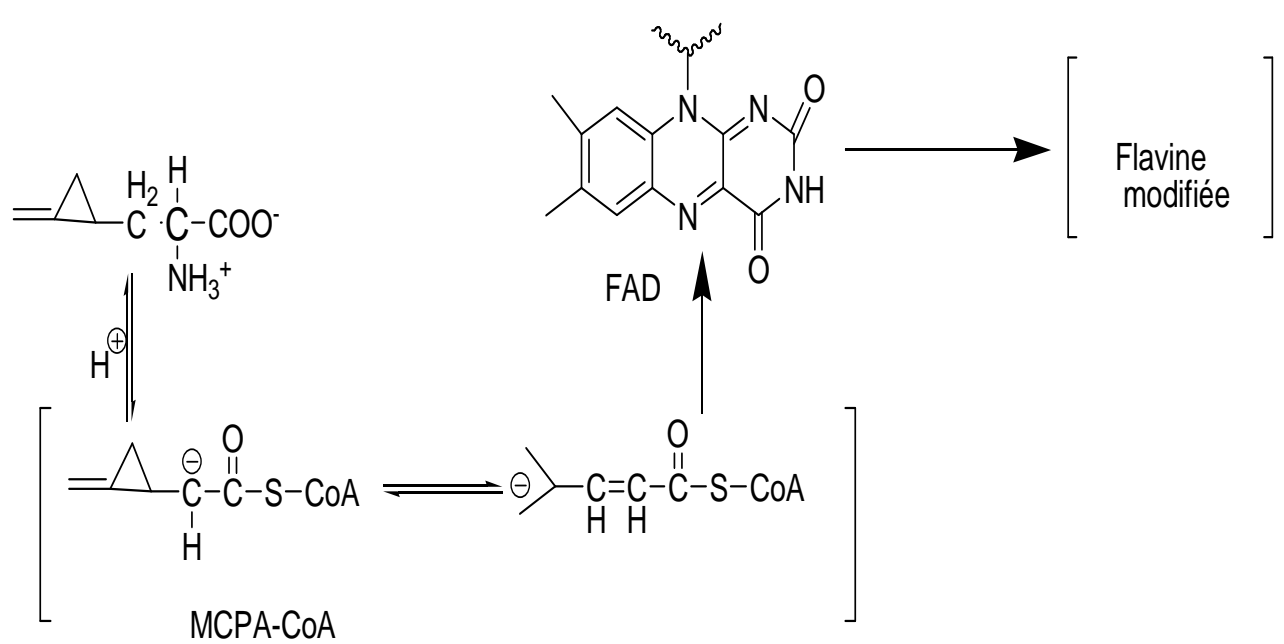

Figure 1 : Mécanisme général d'inactivation de la Flavine Adenine Dinucléotide (FAD) par le Méthylène Cyclopropyl Acétyl CoA (MCPACOA) métabolite actif de l'hypoglycine A selon Alexandra et al. (1981).

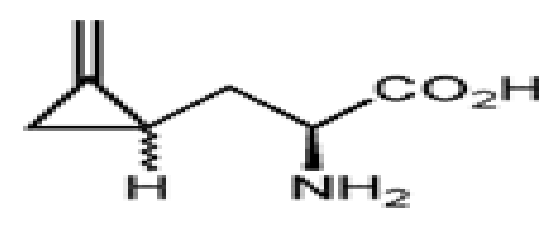

Iypoglycin A<smiles>C=C1C[C@H]1CC(NC(=O)CCC(N)C(=O)O)C(=O)O</smiles>

\section{Hypoglyein B}<smiles>CC1(O)C(=O)C2=C(C(O)CC(=O)C2O)C2CCCCC21</smiles>

Blighinone<smiles>CC1=CC(=O)CC(C)(C)[C@@]1(O)CC[C@H](C)O</smiles>

Vomifoliol 


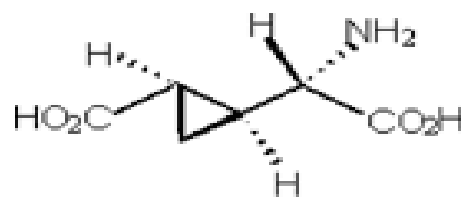

CCG 1

Figure 2 : Structure des molécules isolées de l'arille de BHS (Garg et Mitra, 1968 ; Stuart et al., 1976 ; Kean et Hare, 1980 ; Natalini et al., 2000).

- Récemment, un autre aminoacide non protéinogénique (2S, 1'S, 2 S')-2-(2'carboxycyclopropyl) glycine (CCG1), a été isolé de l'arille de BHS (Natalini et al., 2000). Cette molécule contient comme l'hypoglycine A, le cycle cyclopropane dans sa structure. Notons que le cycle cyclopropane est rare dans la nature ;

- A maturité, l'hypoglycine A passerait de l'arille à la graine en se convertissant en un dipeptide appelé hypoglycine B (Kean et Hare, 1980).

La Figure 2 montre la structure de ces différentes molécules ci-dessus présentées.

\section{CONCLUSION}

Les différents travaux menés sur l'arille de BHS montrent son importance pour l'Homme dans les domaines alimentaire, cosmétique et pharmaceutique. Sa richesse en lipides, protéines, éléments minéraux et en vitamine $\mathrm{C}$ justifie sa valeur nutritionnelle. Cependant, l'arille immature demeure une source d'intoxication redoutable. Aussi, sa consommation à des degrés très élevés, même mûr, aboutirait à des avitaminoses et à des carences nutritionnelles auxquelles les enfants sont les plus vulnérables. Tenant compte de ces facteurs, il faut sensibiliser les populations afin d'éviter les phénomènes morbides.

\section{REFERENCES}

Akintayo ET, Jose CJ, Maria AJ, Gordon CL. 2004. Assessment of dietary exposure to the natural toxin hypoglicin in ackee (Blighia sapida) by Jamaican. Food Research International., 37: 833-838.

Al-bassam SS, Sherratt HSA. 1981. The antagonism of the toxicity of hypoglycin by glycine. Biochem. Pharmac., 30: 2817-2824.

Alexandra W, Colin T, Sandro G. 1981. Inactivation of General Acyl CoA Deshydrogenase Pig Kidney by a metabolite of hypoglicinA. The Journal of Biological Chemistry, 256(19): 98099812.

Arbonnier M. 2002. Arbres, arbustes et lianes des zones sèches d'Afrique de l'ouest. CIRAD- MNHN; 173-179.

Barennes H. Valea I. Boudat A. Idle J. Nagot T. 2004. Early glucose and methylene blue are effective against unripe ackee (Blighia sapida) poisoning in mice. The Journal of Biological Chemistry, 256(19): 9809-9812.

Blancke R. 2001. Guide des Fruits et Légumes Tropicaux. Ulmer; 108.

Brown M, BATES RP, McGowan C, Cornel JA. 1992. Influence of fruit maturity on the hypoglicin A level in ackee (Blighia sapida). Journal of Food Safety, 12(2): 167.

CDC. 1992. Toxic Hypoglycemic Syndrome Jamaica 1989-1991. MMWR January 31, 41(04): 53-55.

Chase GWJ, Landen WOJ, Solimon AG. 1990. Hypoglicin A content in aril, seeds, and husks of ackee fruit at various stages of repenses. Journal of the Association of Official Analytical Chemists, 73: 318- 319.

Djenotin ST, Wotto VD, Lozano P, Pioch D, Sohounhlou DKC. 2009. Characterisation of Blighia sapida (sapindaceae) seed oil and defatted cake from Benin. Natural Product Research, 23(6): 549-560. 
Goldson A. 2011. The ackee fruit (Blighia sapida) and its associated toxic effects. The Science Creative Quaterly, issue 6. http://www.scq.ubc.ca/the-ackee-fruitblighia-sapida-and-its-associated-toxiceffects-2/

Edleston, Person. 2003. Acute plant poisoning and antitoxin antibodies. J Toxicol Clin., 41(3): 309-315.

Food Agriculture Organisation/World Health Organisation. 1998. The application of risk communication to food standards and safety matters. Report of a Joint FAO/WHO Expert Consultation. Rome. 2-6 February 1998.

Food Agriculture Organisation. 2000. Légumineuse, noix et graines oléagineuses. La nutrition dans les pays en développement. F.A.O, Italie.

Food Agriculture Organisation. 2003. Evolution des graisses alimentaires et évolution de leur consommation. Laboratoire de nutrition tropicale, centre ORSTOM 34032, Montpellier cedex, France.

Garg HS, Mitra CR. 1967. Blighia sapida. I. Constituents of the fresh fruit. Plant Med., 13(1): 74-80.

Hassall CH, Reyle K. 1954. Hypoglicin A, B: biologically active polypeptides from Blighia sapida. Nature, 20: 356-357.

Hassall CH, Reyle K. 1955. The toxicity of the ackee (Blighia sapida) and it relationship to the vomiting sickness of Jamaica; a review. West Indian Med J., 4(2): 83-90.

Kean E. 1976. Improved method for isolation of hypoglicin A and B from fruit of Blighia sapida. J. Pharmacol., 26(8): 639-640.

Kean EA, Hare ER. 1980. $\gamma$-Glutamyl transpeptidase of the ackee plant. Phytochemistry., 19: 199-203.

Lewis CB. 1965. Information Bulletin of the Scientific Research Council, 1: 12-14.

Manchester BKL. 1974. Biochemistry of hypoglicin. Febs Letters., 40: s133-s139.

Mcintosh R, Andrews J. 1971. Blighia sapida. Toxic effects on renal morphology and function in rat. NY State J Med., 71(13): 1639-1643.

Meda HA, Diallo B, Buchet JP, Lison D, Barennes H, Ouangre A, Sanou M, Cousens S, Tall F, Van De Perre P. 1999. Epidemic of fatal encephalopathy in preschool childreen in Burkina-Faso and consumption of unripe ackee (Blighia sapida) fruit. Lancet, 353: 536-540.

Ming-tain lai DL, Eugene O, Hung-wen L. 1991. Mechanistic study on the inactivation of general acyl-coA déshydrogénase by a metabolite of hypoglin A. Journal of the American Chemical Society, 113: 7388-7397.

Ming-tain lai DL, Eugene O, Hung-wen L. 1992. Study of the inactivation of general acyl-coA déshydrogénase by (1R) and (1S)- (methylenecyclopropyl) acetyl-coA. Biorganic and Medical Chemistry Letters 2, 1423. Journal of the American Chemical Society, 113: 7388-7397.

Ming-tain lai DL, Eugene O, Hung-wen L. 1993. Inactivation of medium-chain acylcoA déshydrogénase by a metabolite of hypoglicin: characterisation of the major turnover product and evidence suggesting an alternative Flavin modification pathway. Journal of the American Chemical Society, 115: 5.

Morton J., 1987. Akee. In Fruits of Warm Climates, Julia FM (ed). Morton Publisher: Miami, FL; 269-271.

Natalini B, Capodiferro V, De Luca C, Espinal R. 2000. Isolation of pure $(2 \mathrm{~S}$, 1'S, 2 S')-2-(2'- carboxycyclopropyl) glycine from Blighia sapida (Ackee). $J$ Chromatogr A, 873: 283-286.

Oladiji AT, Shoremekun KL, Yakubu MT. 2009. Physicochemical Properties of the Oil Fruit of Blighia sapida and Toxicological Evaluation of the OilBased Diet in Wistar Rats. Journal of Medicinal Food, 12(5): 549-560.

Omobuwajo TO, Sami LA, Olajide JO. 2002. Chemical composition and physicochemical and functional properties of ackee (Blighia sapida) pulp and seed flours. Food Chemistry, 77(3): 333-336. 
Organisation Panamericaine de la Santé. 2002. Empoisonnement par consommation d'ackee (Blighia sapida) dans le Département du Nord, Haïti (Vol. 22, No.2). Organisation Panamericaine de la Santé.

Reeves PG, Nielen FH, Fahey GCJ. 1993. Purified diets for laboratory rodents: final report of the American Institute of Nutrition ad hoc writing committee on the reformulation of the AIN-76A rodent diet. J. Nutr., 123: 1939-1951.

Singh P, Gardner M, Poddar S, Choo-kang E, Coard K, Rickards E. 1992. Toxic effects of ackee oil (Blighia sapida) following subacute administration to rats. Foods Chemical Toxical., 44(2): 207-213.

Stuart KL, Roberts EV, Whittle YG. 1976. Phytochemistry, 15: 332-333.

Tanaka K, Kean EA, Johnson B. 1976. Jamaican vomiting sickness, biochemical investigation of two cases. New England Journal of Medecine, 295(9): 461-467.

Von Holt VHM, Bohm H. 1976. Metabolic effects of methylene-cyclo-propaneacetic acid, a metabolite of hypoglicin. Biochimica and Biophysica Acta., 125(1): 1 . 